assisted study group for the Department of Health, ${ }^{2}$ while yet another group was reviewing local authority health service relationships. ${ }^{3}$ No doubt the working party and the groups had informal contacts but a classic "chicken and egg" dilemma has obviously occurred, and it is small wonder that the Hunter Report is often driven into generalization.

The working party accepts that community physicians should accept "some kind of management responsibility, either as an individual or collectively as a member of a team, for all or some of the services provided." They will not have a purely advisory role. While this will reassure doctors in local authorities or hospitals currently active in administration, the future of those in administration whose main interest is clinical work remains uncertain. In fact the remit of the working party seems to have excluded any study of the 1,000 or more doctors in this group, who make a valuable contribution to the health services. A suitable place for their skills must be found after 1974 .

In their interim report ${ }^{4}$ Dr. Hunter and his colleagues argued the need for retraining doctors at present in medical administration to prepare them for reorganization and the Government has made arrangements for this ${ }^{5}$-though the profession has criticized them as inadequate. ${ }^{6}$ The final report recognizes that if "young doctors of the calibre needed" are to be attracted to community medicine then a better training programme and an attractive career structure are essential.

Though not promoting the claims of community medicine specialists to be "chief executives of the new health service authorities (should such appointments be made)" the working party forecasts that they should be able to exploit the potential benefits of unification "more so perhaps than any other staff. ..."The report stresses the importance of priorities at every level of health services administration and sees the community physician playing a major part in assessing needs and choosing solutions. One of the great benefits of an integrated Service should be a more rational ordering of priorities than is now the case. So his work in trying to equate clinical demands with available resources - a constant source of friction between clinicians and management-could be the community physician's signal contribution to the new N.H.S.

At regional level the chief administrative medical officer, supported by a team of administrative M.O.'s, would be a member of the group of chief officers "bearing the main responsibility for advising the regional authority on the overall development of services." $\mathrm{He}$ and his medical colleagues would also be concerned among other duties with co-ordinating and monitoring area health care services.

At area and district levels the working party is clear about the functions of the community physician but inevitably vague on their deployment. Nevertheless, it favours the "generalist" community physician at district level having a status similar to that of a consultant. Presumably this implies that while the career structure open to a doctor wanting to do administration would compare favourably with that of his clinical colleagues he would not necessarily be called a consultant on reaching career level. He would, however, be part of any district and management team, and the report sees him helping the all important district medical advisory machinery to work effectively.

The report's final-and longest-chapter deals with training and career structure. Lack of time prevented any study of undergraduate training, but with the medical school curriculum already at bursting point this probably does not matter too much. It would be helpful, however, if medical students could at least be encouraged to appreciate the value of good administration. On postgraduate training the report goes into detail and the career structure has obviously been carefully studied.

It is a pity that because of the handicap imposed by the uncertainty of the Government's intentions on reorganization the preciseness of the final chapter could not have applied throughout the report. Nevertheless, despite thisand despite a surfeit of the jargon which disfigures so many official documents-the working party's conclusions should dispel some of the misgivings about 1974 and beyond among medical administrators, particularly local authority doctors. It may also help to reassure clinicians that administration has some good points and show them that their medical colleagues in it are there to help them and their patients.

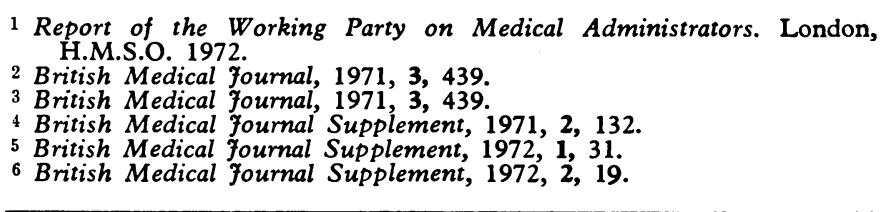

\section{Peripheral Neuropathy and Chronic Liver Diseases}

Peripheral neuropathy may be a complication of diabetes and alcoholism. Lately it has been described in association with a number of other primarily non-neurological diseases. Uraemia is a good example of a condition in which this complication has attracted much interest during the past 10 years. ${ }^{2} 2$ From recent reports it seems possible that chronic liver disease might be added to the group of disorders in which an unknown metabolic derangement may be a cause of neuropathy in some patients. ${ }^{3-6}$

There are several possible causes of peripheral neuropathy in liver diseases. P. K. Thomas and J. G. Walker ${ }^{7}$ described sensory neuropathy in three patients with primary biliary cirrhosis. Nerve biopsies showed xanthomatous deposits in the nerves, so that in these particular cases mechanical derangement of the nerve fibres by the deposits seemed a likely cause of the neuropathy. Peripheral neuropathy is a common complication of alcoholism even in the absence of liver disease. One would therefore except some patients with alcoholic cirrhosis to suffer also from alcoholic neuropathy. Some patients with cirrhosis have diabetes, and neuropathy may occur even in mild diabetes. At our present state of knowledge it is difficult to attribute neuropathy to an independent cause in any patient with diabetes.

If one excludes patients with other possible causes of neuropathy, there remain a group with chronic liver disease of varying aetiology in whom there is some evidence of peripheral neuropathy. R. P. Knill-Jones and colleagues ${ }^{6}$ have described a careful prospective clinical study of $\mathbf{7 0}$ patients with chronic liver disease, of whom 14 were found to have mild peripheral neuropathy. Only two of the 14 patients had any neurological symptoms. Abnormal signs in most instances consisted of depressed tendon reflexes and impaired sense of vibration in the lower limbs. Conduction velocity was mildly impaired. Biopsy studies of the sural nerve at the ankle showed that the neuropathy was of the demyelinating type. The possible aetiological factors were carefully considered and correlations made with many 
aspects of the liver disease. Diabetes was probably a factor in six. In the remaining patients increased levels of $\operatorname{IgA}$ and IgM, oesophageal varices, and a past history of hepatic encephalopathy were common. These associations, together with the observation that neuropathy improved in one patient during treatment with neomycin and a low protein diet, would favour the suggestion that failure of normal metabolic processes is important.

A. M. Davison and colleagues 8 have described uraemic patients in whom the function of the peripheral nerves, as assessed by measurements of conduction velocity, temporarily deteriorated during an intercurrent attack of serum hepatitis. This situation is obviously complex, and is not clear whether disturbed liver metabolism was directly responsible for the impaired nerve function, and whether or not the situation is at all comparable with that in chronic liver disease.

Thus the evidence accumulates that impaired nerve function may occur in liver disease, but severe incapacitating neuropathy has not yet been described. It is unlikely that the metabolic derangement responsible for diabetic, uraemic, and hepatic neuropathy is the same in each instance. Elucidation of the causes of these neuropathies would further our understanding of the functioning of peripheral nerves in health and disease.

1 Asbury, A. K., Victor, M., and Adams, R. D., Archives of Neurology, 1963, $8,413$.

Thomas, P. K., et al., Brain, 1971, 94, 761.

Dayan, A. D., and Williams, R., Lancet, 1967, 2, 133.

- Ortiz Vásquez, J., Olmo, A., Muro, J., Villamor, J., and Barreiro, P., Revista Clinica Española Añda, 1967, 104, 60.

Seneviratne, K. N., and Peiris, O. A., Fournal of Neurology, Neurosurgery and Psychiatry, 1970, 33, 609.

- Knill-Jones, R. P., Goodwill, C. J., Dayan, A. D., and Williams, R., Fournal of Neurology, Neurosurgery and Psychiatry, 1972, 35, 22.

7 Thomas, P. K., and Walker, J. G., Brain, 1965, 88, 1079.

- Davison, A. M., Williams, I. R., Mawdsley, C., and Robson, J. S., British Medical fournal, 1972, 1, 409.

\section{Febrile Convulsions in Early Childhood}

Childhood convulsions associated with fever are commonly difficult to diagnose. Convulsions are common in childhood, affecting some $6 \%$ of children in the first five years, and it may be helpful to rehearse the salient facts about them.1-3

When a child has a fit, and is found to have a raised temperature, he may have a "febrile convulsion." This has a good prognosis and is most unlikely to be followed by epilepsy in later years. ${ }^{12}$ The title is unfortunate and may itself be one of the main sources of confusion. To fall into this category with a good prognosis the child should be not less than 6 months and not over 5 or possibly 6 years of age; the fit should occur only with the rapid rise of temperature, and should not be repeated with the same infection; there should be a clear history that the child was unwell at least for an hour or two before the convulsion occurred; the fit should not last more than 10 minutes; it should not be focal, and there should be no Todd's paralysis (post- epileptic hemiplegia) after it; and there must be no history of fits occurring without a rapid rise of temperature. A family history of febrile convulsions is common-more common than a family history of epilepsy in epileptic children. A definite family history of epilepsy would make the diagnosis of febrile convulsions uncertain. Febrile convulsions are commonly caused by otitis media, tonsillitis, pneumonia, and other common infections. The electroencephalogram after the infection has subsided is normal.

Fever may precipitate fits in epileptics: hence the importance of adhering to the criteria given above. In particular, for a diagnosis of "febrile convulsion" there must be no history of fits occurring without a rapid rise of temperature. Severe convulsions cause a rise of temperature, ${ }^{4}$ so that the finding of a raised temperature when a child has had a fit does not prove that he has an infection.

The convulsion may be the result of intracranial disease and not the result of the fever. Hence many paediatricians always perform a lumbar puncture to exclude the possibility of a pyogenic meningitis. The so-called acute infantile hemi- i plegia is manifested often by fever with prolonged convul- O্ sions followed by neurological sequelae, and is due to a $\vec{\nexists}$ variety of causes. It is partly this condition which has caused 8 some authors to express the idea that "febrile convulsions" have a serious prognosis, ${ }^{5}$ but it does not satisfy the criteria laid down by $\mathrm{S}$. Livingston ${ }^{12}$ and others for the diagnosis of "febrile convulsions."

The precise cause of the fit in febrile convulsions is not understood. It is known that kittens, but not cats, when ex- $\vec{\varphi}$ posed to a rapid rise of temperature may have convulsions, $N$ and it is only the young child, under the age of 5 or 6 , who responds in this way. Provided the criteria for diagnosis are adhered to there is nothing in the previous history to suggest that children who do have febrile convulsions differ from children who do not.

When a child has a febrile convulsion, as a first-aid measure he should be tepid sponged or placed in a cool bath-but not a hot bath. Parents must be dissuaded from determined efforts to "warm him up" by putting him in a hot bath or overclothing him. If it is available, intramuscular paraldehyde should be given and the child sent immediately to hospital. Continuous prophylactic antiepileptic treatment should not be given: it is unnecessary and unhelpful. When the child has his next rise of temperature, the mother should take immediate steps to reduce his temperature by tepid sponging and giving aspirin. The child should also receive phenytoin or phenobarbitone or both, $\sigma$ but only for 24 hours, for the danger of a fit is only when there is a rapid rise of temperature. The mother should have a small supply at hand, for the child will have further infections. After the age of 5 or 6 the danger of febrile convulsions is at an end.

\footnotetext{
Livingston, S., Bridge, E. M., and Kajdi, L., Fournal of Pediatrics, 1947, 31, 509.

Livingston, S., The Diagnosis and Treatment of Convulsive Disorders in Children. Springfield, Charles Thomas, 1954.

3 Illingworth, R. S., Common Symptoms of Disease in Children. Oxford, Blackwell, 1971 .

Lennox, W. G., Pediatrics, 1953, 11, 341.

- Peterman, M. G., fournal of Pediatrics, 1952, 41, 536.
} . 飬 . 